\title{
Magnetic Inhomogeneities in 3D Ferromagnetic spin system
}

\author{
G. Addaline Stally and M. M. Latha \\ Department of Physics, Women's Christian College, Nagercoil, \\ Affiliated to Manonmaniam Sundaranar University, Abishekapatti, Tirunelveli- 627012, \\ India.
}

\begin{abstract}
We investigate the magnetization dynamics of three dimensional inhomogeneous ferromagnetic spin system by proposing a model Hamiltonian incorporating bilinear interactions with site dependent inhomogeneity. The dynamics is represented by a $(3+1)$ dimensional Perturbed Nonlinear Schrödinger Equation (PNLSE). The effect of inhomogeneity is understood by carrying out Sine-Cosine function analysis. Here six types of magnetic inhomogeneities have been revealed. The range of values of the parameters which determine the possibility of existence of each of these inhomogeneities have been determined. An interesting dynamical scenario where the overtaking nature of solitons is observed.
\end{abstract}

\section{Introduction}

Magnetic inhomogenity and soliton study is a recent field of investigation and of great importance in fundamental physics as well as in different research areas [1-3]. Such magnetic inhomogeneity occurs in real magnetic materials due to the appearance of various magnetic defects such as imperfect grain boundaries, impurities as well as elastic defects such as misfitting precipitates and dislocations. The presence of such perturbation can lead to the formation of different kinds of magnetic inhomogeneities which influence the process of the magnetization reversal of a sample [4,5]. Moreover, the propagation of solitons in the presence of inhomogeneities concerns an important physical property of magnetic systems. A soliton in an integrable system moves with constant shape and velocity, but in realistic because of the inhomogeneity of the magnetic materials, solitons may exhibit more complex motion with changing velocity and shape which may be used as a desirable effect for fast transport and fast communication [6,7]. Thus inhomogeneity has a significant effect on the magnetization dynamics of the ferromagnet.

It is also very interesting to study the effect of an inhomogeneity in the system modeled by $(3+1)$ dimensional heisenberg spin equation. Some pioneering and fundamental work of inhomogeneous FM spin chain in one dimensional case has been widely researched during the past few decades[6-10]. Followed by that there are also studies on two dimensional inhomogeneous ferromagnetic system [11,12]. Recently the present authors have reported an integrable model of 3D ferromagnetic spin system [13] taking into account only the bilinear and anisotropic interactions. However the nonlinear excitation of 3-dimensional FM spin system with inhomogeneities has not yet been analysed.

The aim of the present study is to construct a series of exact solitary wave solutions of a PNLSE governing a three dimensional Heisenberg spin system. We solve the $(3+1)$ dimensional PNLSE by employing the Sine-Cosine method embedded with symbolic computation. The paper is organized as follows. In Section 2, we formulate the Hamiltonian for the inhomogenous 3D ferromagnetic spin system with bilinear and anisotropic interactions. In Section 3, we implement the perturbation technique to solve the lower order PNLSE with site dependent inhomogeneity and bilinear interactions. In Section 4, we discuss the effect of inhomogeneity and conclude the results in section 5. 


\section{Inhomogeneous Ferromagnetic spin system: Model Hamiltonian}

In order to investigate the dynamical properties of an inhomogeneous lattice described by the 3D ferromagnetic spin system, we write the Hamiltonian with the site dependent inhomogeneity $f_{i, j . k}$ in the form

$$
\begin{aligned}
& \widetilde{H}=-\sum_{i, j, k}\left\{f _ { i , j , k } \left[\tilde{J}\left(\vec{S}_{i, j, k} \cdot \vec{S}_{i+1, j, k}\right)+\tilde{J}^{\prime}\left(\vec{S}_{i, j, k} \cdot \vec{S}_{i, j+1, k}\right)+\tilde{J}^{\prime \prime}\right.\right. \\
& \left(\vec{S}_{i, j, k} \cdot \vec{S}_{i, j, k+1}\right)+\tilde{J}_{1}\left(\vec{S}_{i, j, k} \cdot \vec{S}_{i+1, j+1, k}\right)+\tilde{J}_{1}^{\prime}\left(\vec{S}_{i, j, k} \cdot \vec{S}_{i+1, j, k+1}\right) \\
& \left.\left.+\tilde{J}_{1}^{\prime \prime}\left(\vec{S}_{i, j, k} \cdot \vec{S}_{i, j+1, k+1}\right)+\tilde{J}_{2}\left(\vec{S}_{i, j, k} \cdot \vec{S}_{i+1, j+1, k+1}\right)\right]-\tilde{A}\left(\vec{S}_{i, j, k}^{z}\right)^{2}\right] .
\end{aligned}
$$

In Eq.(1), $f_{i, j, k}$ characterizes the inhomogeneity in bilinear interaction and $J, J^{\prime}, J^{\prime \prime}$, $J_{1}, J_{1}^{\prime}, J_{1}^{\prime \prime}, J_{2}$ correspond to bilinear exchange interactions for ferromagnetic system with parallel spins and $A$ is the uniaxial anisotropy interactions of lower order. To transform the spin Hamiltonian into its dimensionless form, we replace $\vec{S}_{i, j, k}=\hbar \hat{S}_{i, j, k}$ and $\hat{S}_{i, j, k}^{ \pm}=\hat{S}_{i, j, k}^{x} \pm$ $\hat{S}_{i, j, k}^{y}$. After introducing $H=\frac{\tilde{H}}{\hbar^{2} S^{2}}$ and $A=\tilde{A}$, the dimensionless Hamiltonian with the inhomogeneity can be written as

$$
\begin{aligned}
& H=-\sum_{i, j, k}\left\{f _ { i , j , k } \left[\frac{J}{2 S^{2}}\left(\hat{S}_{i, j, k}^{+} \cdot \hat{S}_{i+1, j, k}^{-}+\hat{S}_{i, j, k}^{-} \cdot \hat{S}_{i+1, j, k}^{+}+2 \hat{S}_{i, j, k}^{z} \cdot \hat{S}_{i+1, j, k}^{z}\right)+\right.\right. \\
& \frac{J^{\prime}}{2 S^{2}}\left(\hat{S}_{i, j, k}^{+} \cdot \hat{S}_{i, j+1, k}^{-}+\hat{S}_{i, j, k}^{-} \cdot \hat{S}_{i, j+1, k}^{+}+2 \hat{S}_{i, j, k}^{z} \cdot \hat{S}_{i, j+1, k}^{z}\right)+\frac{J^{\prime \prime}}{2 S^{2}}\left(\hat{S}_{i, j, k}^{+} \cdot\right. \\
& \left.\hat{S}_{i, j, k+1}^{-}+\hat{S}_{i, j, k}^{-} \cdot \hat{S}_{i, j, k+1}^{+}+2 \hat{S}_{i, j, k}^{z} \cdot \hat{S}_{i, j, k+1}^{z}\right)+\frac{J_{1}}{2 S^{2}}\left(\hat{S}_{i, j, k}^{+} \cdot \hat{S}_{i+1, j+1, k}^{-}+\right. \\
& \left.\hat{S}_{i, j, k}^{-} \cdot \hat{S}_{i+1, j+1, k}^{+}+2 \hat{S}_{i, j, k}^{z} \cdot \hat{S}_{i+1, j+1, k}^{z}\right)+\frac{J_{1}^{\prime}}{2 S^{2}}\left(\hat{S}_{i, j, k}^{+} \cdot \hat{S}_{i+1, j, k+1}^{-}+\hat{S}_{i, j, k}^{-} \cdot\right. \\
& \left.\hat{S}_{i+1, j, k+1}^{+}+2 \hat{S}_{i, j, k}^{z} \cdot \hat{S}_{i+1, j, k+1}^{z}\right)+\frac{J_{1}^{\prime \prime}}{2 S^{2}}\left(\hat{S}_{i, j, k}^{+} \cdot \hat{S}_{i, j+1, k+1}^{-}+\hat{S}_{i, j, k}^{-} \cdot\right. \\
& \left.\hat{S}_{i, j+1, k+1}^{+}+2 \hat{S}_{i, j, k}^{z} \cdot \hat{S}_{i, j+1, k+1}^{z}\right)+\frac{J_{2}}{2 S^{2}}\left(\hat{S}_{i, j, k}^{+} \cdot \hat{S}_{i+1, j+1, k+1}^{-}+\hat{S}_{i, j, k}^{-} .\right. \\
& \left.\left.\hat{S}_{i+1, j+1, k+1}^{+}+2 \hat{S}_{i, j, k}^{z} \cdot \hat{S}_{i+1, j+1, k+1}^{z}\right)-\frac{A}{S^{2}}\left(\hat{S}_{i, j, k}^{z}\right)^{2}\right] .
\end{aligned}
$$

Since we have to bosonize the Hamiltonian in semiclassical level we use Holstein-Primakoff (H-P) [14] transformation which expresses spin operator in terms of the boson creation and anihilation operators as:

$$
\begin{aligned}
\hat{S}_{i, j, k}^{+} & =\sqrt{2}\left[1-\varepsilon^{2} a_{i, j, k}^{\dagger} a_{i, j, k}\right]^{\frac{1}{2}} \varepsilon a_{i, j, k}, \\
\hat{S}_{i, j, k}^{-} & =\sqrt{2} \varepsilon a_{i, j, k}^{\dagger}\left[1-\varepsilon^{2} a_{i, j, k}^{\dagger} a_{i, j, k}\right]^{\frac{1}{2},} \\
\hat{S}_{i, j, k}^{z} & =\left[1-\varepsilon^{2} a_{i, j, k}^{\dagger} a_{i, j, k}\right],
\end{aligned}
$$

where $\varepsilon=\left(\frac{1}{S}\right)^{\frac{1}{2}}$ is a dimensionless parameter. The bosonic operators $a_{i, j, k}, a_{i, j, k}^{\dagger}$ satisfy the usual bose commutations relations as $\left[a_{m}, a_{n}^{\dagger}\right]=\delta_{m n}$ and $\left[a_{m}, a_{n}\right]=\left[a_{m}^{\dagger}, a_{n}^{\dagger}\right]=0$. We use the semiclassical expansions in the following form:

$$
\begin{aligned}
& \frac{\hat{S}_{i, j, k}^{+}}{S}=\sqrt{2}\left[1-\frac{\varepsilon^{2}}{4} a_{i, j, k}^{\dagger} a_{i, j, k}-O\left(\varepsilon^{4}\right)\right] \varepsilon a_{i, j, k}, \\
& \frac{\hat{S}_{i, j, k}^{-}}{S}=\sqrt{2} \varepsilon a_{i, j, k}^{\dagger}\left[1-\frac{\varepsilon^{2}}{4} a_{i, j, k}^{\dagger} a_{i, j, k}-O\left(\varepsilon^{4}\right)\right] .
\end{aligned}
$$

The spin operators can be expressed in power series of $\varepsilon$ by using Eqs.(6) and (7) as 
where

$$
H=D_{0}^{\prime}+\varepsilon^{2} D_{1}^{\prime}+\varepsilon^{4} D_{2}^{\prime},
$$

$$
\begin{aligned}
& D_{0}^{\prime}=\left(A-J-J^{\prime}-J^{\prime \prime}-J_{1}-J_{1}^{\prime}-J_{1}^{\prime \prime}-J_{2}\right) f_{i, j, k}, \\
& D_{1}^{\prime}=\left(E_{0}+J E_{1}+J^{\prime} E_{2}+J^{\prime \prime} E_{3}+J_{1} E_{4}+J_{1}^{\prime} E_{5}+J_{1}^{\prime \prime} E_{6}+J_{2} E_{7}\right), \\
& D_{2}^{\prime}=\left(K_{0}+J K_{1}+J^{\prime} K_{2}+J^{\prime \prime} K_{3}+J_{1} K_{4}+J_{1}^{\prime} K_{5}+J_{1}^{\prime \prime} K_{6}+J_{2} K_{7}\right) . E_{i}, K_{i}, i=0,1 \ldots 7 \text { are }
\end{aligned}
$$
given in Appendix A.

The spin dynamics can be expressed in terms of the Heisenberg equation of motion for the boson operators by substituting Hamiltonian (8) in the following equation of motion.

$$
i \hbar \frac{\partial a_{i, j, k}}{\partial t}=\left[a_{i, j, k}, H\right]
$$

Using Glauber Coherent representation [15] for bose operators as $\langle u| a_{i, j, k}^{\dagger}=\langle u| a_{i, j, k}^{*}$ and $a_{i, j, k}|u\rangle=u_{i, j, k}|u\rangle$ where $|u\rangle=\Pi_{i, j, k}\left|u_{i, j, k}\right\rangle$ with $\langle u \mid u\rangle=1$, where $u_{i, j, k}$ is the coherent state wave function and the dynamics of 3D inhomogeneous FM spin system can be expressed by the following equation of motion for the average $\left\langle u\left|a_{i, j, k}\right| u\right\rangle$ as

where

$$
i \hbar \frac{\partial u_{i, j, k}}{\partial t}-\varepsilon^{2} F_{0}^{\prime}-\frac{\varepsilon^{4}}{4} F_{1}^{\prime}=0
$$

$$
\begin{aligned}
& F_{0}^{\prime}=Q_{0}+J Q_{1}+J^{\prime} Q_{2}+J^{\prime \prime} Q_{3}+J_{1} Q_{4}+J_{1}^{\prime} Q_{5}+J_{1}^{\prime \prime} Q_{6}+J_{2} Q_{7}, \\
& F_{1}^{\prime}=R_{0}+J R_{1}+J^{\prime} R_{2}+J^{\prime \prime} R_{3}+J_{1} R_{4}+J_{1}^{\prime} R_{5}+J_{1}^{\prime \prime} R_{6}+J_{2} R_{7} . Q_{i}, R_{i}, i=0,1 \ldots 7 \text { are }
\end{aligned}
$$
given in Appendix $B$.

Eq.(10) describes the nonlinear spin dynamics of inhomogeneous $(3+1)$ dimensional ferromagnet. In the semiclassical limit, one can approximate the discrete spin problem by using the general continuum expansions as

$$
F^{(n)}(t)=\sum_{m=0}^{n} n_{m} \frac{\partial^{n} f}{\partial x^{m} \partial y^{n-m}}\left(x_{0}+t \Delta x, y_{0}+t \Delta y\right) \Delta x^{m} \Delta y^{n-m},
$$

where $n_{m}=\frac{n !}{m !(n-m) !}$ is the standard binomial coefficient.

Thus the final form of continuum equation of motion representing the spin dynamics of 3D inhomogeneous FM spin system with bilinear and anisotropy interaction is found to be

$$
\begin{aligned}
& i u_{t}+m_{1} u_{x x}+m_{2} u_{y y}+m_{3} u_{z z}+m_{4} u_{x y}+m_{5} u_{x z}+m_{6} u_{y z}+m_{7} u_{y x} \\
& \quad+m_{8} u_{z y}+m_{9} u_{z x}+m_{10} u_{x}+m_{11} u_{y}+m_{12} u_{z}+m_{13} u-m_{14}|u|^{2} u=0,
\end{aligned}
$$

where $m_{1}=\gamma^{4}\left(J+J_{1}+J_{2}+J_{1}^{\prime}\right) f ; m_{2}=\gamma^{4}\left(J_{1}+J_{2}+J_{1}^{\prime}+J_{1}^{\prime \prime}\right) f ; m_{3}=\gamma^{4}\left(J_{2}+J^{\prime \prime}+J_{1}^{\prime}+\right.$ $\left.J_{1}^{\prime \prime}\right) f ; m_{4}=\gamma^{4}\left(J_{1}+J_{2}\right) f ; m_{5}=\gamma^{4}\left(J_{2}+J_{1}^{\prime}\right) f ; m_{6}=\gamma^{4}\left(J_{2}+J_{1}^{\prime \prime}\right) f ; m_{7}=\gamma^{4}\left(J_{1}\right) f ; m_{8}=$ $\gamma^{4}\left(J_{1}^{\prime \prime}\right) f \quad ; \quad m_{9}=\gamma^{4}\left(J_{1}^{\prime}\right) f \quad ; \quad m_{10}=\gamma^{4}\left(f_{x} J+f_{x} J_{1}+f_{x} J_{2}+f_{x} J_{1}^{\prime}+f_{y} J_{1}+f_{y} J_{2}+f_{z} J_{2}+\right.$ $\left.f_{z} J_{1}^{\prime}\right) ; m_{11}=\gamma^{4}\left(f_{x} J_{1}+f_{x} J_{2}+f_{y} J_{1}+f_{y} J_{2}+f_{y} J^{\prime}+f_{y} J_{1}^{\prime \prime}+f_{z} J_{2}+f_{z} J_{1}^{\prime \prime}\right) ; m_{12}=\gamma^{4}\left(f_{x} J_{2}+\right.$ $\left.f_{x} J_{1}^{\prime}+f_{y} J_{2}+f_{y} J_{1}^{\prime \prime}+f_{z} J_{2}+f_{z} J_{1}^{\prime}+f_{z} J^{\prime \prime}+f_{z} J_{1}^{\prime \prime}\right) ; m_{13}=2 \gamma^{2} A ; m_{14}=2 \gamma^{4} A$.

Eq.(12) is a $(3+1)$ dimensional PNLSE. In order to gain deeper understanding on the PNLSE, we make an attempt to solve Eq.(12) using Sine-Cosine function method which is explained in the following section. 


\section{Nonlinear Excitations}

In this section we employ the Sine-Cosine function method $[16,17]$ to solve PNLSE (12). This method is used to obtain the exact solutions for different types of nonlinear partial differential equations. To use this method, first we make the transformation $u=Q e^{i \theta}$ where $\theta=a_{1} x+a_{2} y+a_{3} z+a_{4} t$ in Eq.(12) and separate the real and imaginary parts to get

$$
\begin{aligned}
& i Q_{t}-a_{4} Q+m_{1}\left(Q_{x x}+2 i a_{1} Q_{x}-a_{1}^{2} Q\right)+m_{2}\left(Q_{y y}+2 i a_{2} Q_{y}-a_{2}^{2} Q\right) \\
& +m_{3}\left(Q_{z z}+2 i a_{2} Q_{z}-a_{3}^{2} Q\right)+m_{4}\left(Q_{x y}+i a_{1} Q_{y}+i a_{2} Q_{x}-a_{1} a_{2} Q\right) \\
& +m_{5}\left(Q_{x z}+i a_{1} Q_{z}+i a_{2} Q_{x}-a_{1} a_{3} Q\right)+m_{6}\left(Q_{y z}+i a_{2} Q_{y}+i a_{3} Q_{x}\right. \\
& \left.-a_{2} a_{3} Q\right)+m_{7}\left(Q_{y x}+i a_{2} Q_{y}+i a_{1} Q_{x}-a_{2} a_{1} Q\right)+m_{8}\left(Q_{z y}+i a_{3} Q_{y}\right. \\
& \left.+i a_{2} Q_{z}-a_{3} a_{2} Q\right)+m_{9}\left(Q_{z x}+i a_{3} Q_{x}+i a_{1} Q_{z}-a_{3} a_{1} Q\right)+m_{10}\left(Q_{x}+\right. \\
& \left.i a_{1} Q\right)+m_{11}\left(Q_{y}+i a_{2} Q\right)+m_{12}\left(Q_{z}+i a_{3} Q\right)+m_{13} Q-m_{14} Q^{3}=0 . \\
& \\
& -a_{4} Q+m_{1}\left(Q_{x x}-a_{1}^{2} Q\right)+m_{2}\left(Q_{y y}-a_{2}^{2} Q\right)+m_{3}\left(Q_{z z}-a_{2}^{2} Q\right) \\
& +m_{4}\left(Q_{x y}-a_{1} a_{2} Q\right)+m_{5}\left(Q_{x z}-a_{1} a_{3} Q\right)+m_{6}\left(Q_{y z}-a_{2} a_{3} Q\right) \\
& +m_{7}\left(Q_{y x}-a_{2} a_{1} Q\right)+m_{8}\left(Q_{z y}-a_{3} a_{2} Q\right)+m_{9}\left(Q_{z x}-a_{3} a_{1} Q\right) \\
& +m_{10} Q_{x}+m_{11} Q_{y}+m_{12} Q_{z}+m_{13} Q-m_{14} Q^{3}=0
\end{aligned}
$$

and

$$
\begin{aligned}
& Q_{t}+2 m_{1} a_{1} Q_{x}+2 m_{2} a_{2} q_{y}+2 m_{3} a_{3} q_{z}+m_{4}\left(a_{1} q_{y}+a_{2} q_{x}\right) \\
& +m_{5}\left(a_{1} Q_{z}+a_{3} Q_{x}\right)+m_{6}\left(a_{2} Q_{z}+a_{3} Q_{y}\right)+m_{7}\left(a_{2} Q_{x}+a_{1} Q_{y}\right) \\
& +m_{8}\left(a_{3} Q_{y}+a_{2} Q_{z}\right)+m_{9}\left(a_{3} Q_{z}+a_{1} Q_{z}\right)+m_{10} a_{1} Q \\
& +m_{11} a_{2} Q+m_{12} a_{3} Q=0 .
\end{aligned}
$$

To find the travelling wave solution of Eq.(13), we introduce the wave variable $\xi=x+y+$ $z-c t$. Eqs.(14) and (15) now becomes

$$
\begin{aligned}
& -a_{4} Q+m_{1}\left(q_{\xi \xi}-a_{1}^{2} Q\right)+m_{2}\left(q_{\xi \xi}-a_{2}^{2} Q\right)+m_{3}\left(q_{\xi \xi}-a_{3}^{2} Q\right) \\
& +m_{4}\left(q_{\xi \xi}-a_{1} a_{2} Q\right)+m_{5}\left(q_{\xi \xi}-a_{1} a_{3} Q\right)+m_{6}\left(q_{\xi \xi}-a_{2} a_{3} Q\right) \\
& +m_{7}\left(q_{\xi \xi}-a_{2} a_{1} Q\right)+m_{8}\left(q_{\xi \xi}-a_{3} a_{2} Q\right)+m_{9}\left(q_{\xi \xi}-a_{3} a_{1} Q\right) \\
& +m_{10} q_{\xi}+m_{11} q_{\xi}+m_{12} q_{\xi}+m_{13} Q-m_{14} Q^{3}=0
\end{aligned}
$$

and

$$
\begin{aligned}
& -c q_{\xi}+2 m_{1} a_{1} Q_{\xi}+2 m_{2} a_{2} Q_{\xi}+2 m_{3} a_{3} Q_{\xi}+m_{4}\left(a_{1} Q_{\xi}+a_{2} q_{\xi}\right) \\
& +m_{5}\left(a_{1} Q_{\xi}+a_{3} Q_{\xi}\right)+m_{6}\left(a_{2} Q_{\xi}+a_{3} Q_{\xi}\right)+m_{7}\left(a_{2} Q_{\xi}+a_{1} Q_{\xi}\right) \\
& +m_{8}\left(a_{3} Q_{\xi}+a_{2} Q_{\xi}\right)+m_{9}\left(a_{3} Q_{\xi}+a_{1} Q_{\xi}\right)+m_{10} a_{1} Q+m_{11} a_{2} Q \\
& +m_{12} a_{3} Q=0 .
\end{aligned}
$$

The solution of Eqs.(16) and (17) is assumed to be

$$
Q(\xi)=\lambda \cos ^{\beta}(\mu \xi)
$$

where $\lambda, \beta$ and $\mu$ are constant parameters to be determined. To find $\beta$, we balance the higher order derivative term with nonlinear terms of the evolution equations which gives $\beta$ $=-1$. Further substituting the value of $\beta$ in Eqs.(16) and (17), we obtain a system of algebraic equations:

$$
\begin{aligned}
& \cos s^{-1}(\mu \xi):-a_{4} \lambda-m_{1} \mu^{2} \lambda-m_{1} a_{1}^{2} \lambda-m_{2} \mu^{2} \lambda-m_{2} a_{2}^{2} \lambda \\
& -m_{3} \mu^{2} \lambda-m_{3} a_{3}^{2} \lambda-m_{4} \mu^{2} \lambda-m_{4} a_{1} a_{2} \lambda
\end{aligned}
$$




$$
\begin{aligned}
& -m_{5} \mu^{2} \lambda-m_{5} a_{1} a_{3} \lambda-m_{6} \mu^{2} \lambda-m_{6} a_{2} a_{3} \lambda \\
& -m_{7} \mu^{2} \lambda-m_{7} a_{2} a_{1} \lambda-m_{8} \mu^{2} \lambda-m_{8} a_{3} a_{2} \lambda \\
& -m_{9} \mu^{2} \lambda-m_{9} a_{3} a_{1} \lambda+m_{13} \lambda=0, \\
& \cos s^{-3}(\mu \xi): 2 m_{1} \mu^{2} \lambda+2 m_{2} \mu^{2} \lambda+2 m_{3} \mu^{2} \lambda+2 m_{4} \mu^{2} \lambda \\
& +2 m_{5} \mu^{2} \lambda+2 m_{6} \mu^{2} \lambda+2 m_{7} \mu^{2} \lambda+2 m_{8} \mu^{2} \lambda \\
& +2 m_{9} \mu^{2} \lambda-m_{13} \lambda^{3}=0, \\
& \cos s^{-2}(\mu \xi) \sin (\mu \xi): m_{10} \mu \lambda+m_{11} \mu \lambda+m_{12} \mu \lambda=0, \\
& \cos s^{-1}(\mu \xi): m_{10} a_{1} \lambda+m_{11} a_{2} \lambda+m_{12} a_{3} \lambda=0, \\
& \cos ^{-2}(\mu \xi) \sin _{3}(\mu \xi):-c \mu \lambda+2 m_{1} a_{1} \mu \lambda+2 m_{2} a_{2} \mu \lambda+2 m_{3} a_{3} \mu \lambda \\
& +m_{4} a_{1} \mu \lambda+m_{4} a_{2} \mu \lambda+m_{5} a_{1} \mu \lambda+m_{5} a_{3} \mu \lambda \\
& +m_{6} a_{2} \mu \lambda+m_{6} a_{3} \mu \lambda+m_{7} a_{2} \mu \lambda+m_{7} a_{1} \mu \lambda \\
& +m_{8} a_{3} \mu \lambda+m_{8} a_{2} \mu \lambda+m_{9} a_{3} \mu \lambda+m_{9} a_{1} \mu \lambda=0 .
\end{aligned}
$$

The above equations are solved with the aid of symbolic computation and the values of the parameters are found to be

$$
\mu=\sqrt{\frac{-a_{4}+m_{0}+A^{\prime}}{m_{1}+m_{2}+m_{3}+m_{4}+m_{5}+m_{6}+m_{7}+m_{8}+m_{9}}}
$$

where $\quad A^{\prime}=-a_{1}^{2} m_{1}-a_{2}^{2} m_{2}-a_{3}^{2} m_{3}-a_{1} a_{2} m_{4}-a_{1} a_{3} m_{5}-a_{2} a_{3} m_{6}-a_{1} a_{2} m_{7}-$ $a_{2} a_{3} m_{8}-a_{1} a_{3} m_{9}$ and

with

$$
\lambda=\frac{1}{\sqrt{m_{14}}}\left\{\sqrt{\mu\left(2 s_{1}+s_{2}+s_{3}-c\right)+\mu^{2}\left(2 s_{4}+s_{5}\right)}\right\},
$$

$$
\begin{aligned}
s_{1}=a_{1} m_{1}+ & a_{2} m_{2}+a_{3} m_{3}, s_{2} \\
& =\left(a_{1}+a_{2}\right) m_{4}+\left(a_{1}+a_{3}\right) m_{5}+\left(a_{2}+a_{3}\right) m_{6}+\left(a_{2}+a_{1}\right) m_{7}+\left(a_{3}\right. \\
& \left.+a_{2}\right) m_{8}+\left(a_{3}+a_{1}\right) m_{9}, s_{3}=m_{10}+m_{11}+m_{12}, s_{4} \\
& =m_{1}+m_{2}+m_{3}+m_{4}+m_{5}+m_{6}+m_{7}+m_{8}+m_{9}, s_{5} \\
& =a_{1} m_{10}+a_{2} m_{11}+a_{3} m_{12} .
\end{aligned}
$$

The solution of Eq.(12) is then expressed as

$$
\begin{aligned}
& u(x, y, z, t)=\frac{1}{\sqrt{m_{14}}}\left\{\sqrt{\mu\left(2 s_{1}+s_{2}+s_{3}-c\right)+\mu^{2}\left(2 s_{4}+s_{5}\right)}\right\} \\
& \times \sec h\left[\sqrt{\frac{-a_{4}+m_{0}+A}{m_{1}+m_{2}+m_{3}+m_{4}+m_{5}+m_{6}+m_{7}+m_{8}+m_{9}}}\right. \\
& \times(x+y+z-c t)] e^{i\left(a_{1} x+a_{2} y+a_{3} z+a_{4} t\right)} .
\end{aligned}
$$

\section{Effect of inhomogeneity}

In this section, we describe the shape changing behaviour of magnetic soliton under the influence of inhomogeneity. Here we analyse a series of exact propagating soliton solutions for the following types of inhomogeneities such as (i) Linear (ii) Localized (iii) Cubic (iv) Quadratic (v) Periodic and (vi) Bi-quadratic type inhomogeneies. $f=1$ corresponds to homogenous case which supports a stable unperturbed soliton travelling with constant velocity. It is described in Figure 1, for the parameters $J=13.6, J^{\prime}=3.6, J^{\prime \prime}=6$, $J_{1}=6, \zeta_{1}^{\prime}=16, J_{1}^{\prime \prime}=2.7, J_{2}=16, A=0.1, \gamma=0.6, a_{1}=a_{2}=a_{3}=1, a_{4}=0.3$, $y=0.0001$ and $z_{1}=0.11$. 


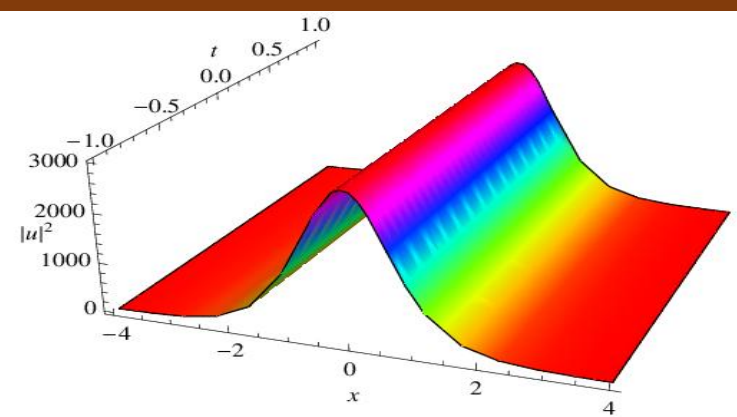

Figure 1: Unperturbed soliton for homogenous system with $f=1$.

The first choice of inhomogeneity is a linear one of the form $f=1+P_{1} x$ that can arise from the three dimensional lattice deformation. For $P_{1} \leq 1$, a single soliton transmission is noticed. When the inhomogeneity parameters exceed 1 , one soliton starts splitting into two solitons which collide with each other as time goes on retaining its shape and amplitude after collision. As $P_{1}$ is further increased, the amplitude found to increase drastically which is portrayed in Figure 2(b). Figures 2(c)-2(e) show the evolution of the overtaking interaction between two separate solitons. Similar behaviour is observed in the case of localized type of inhomogeneity of the form $f=1+P_{2} * \tan h(x)$ which is shown in Figure 3.
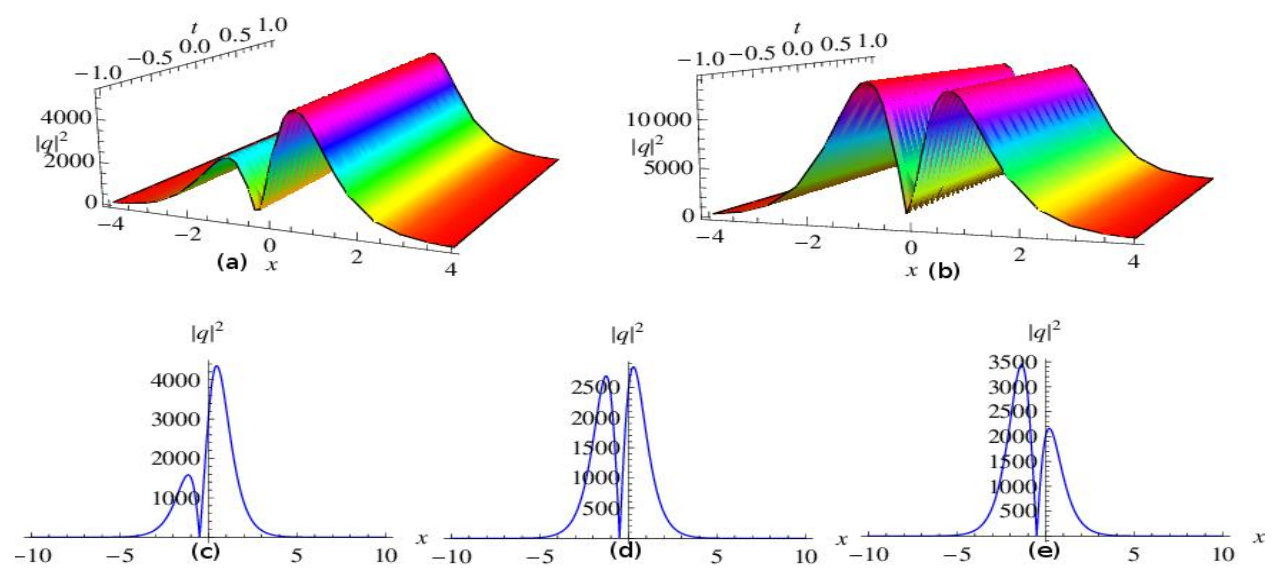

Figure 2: Soliton solution for linear inhomogeneity with a) $P_{1}=2.8$ b) $P_{1}=10$ c) $t=0$ d) $t=-37$ and e) $t=-57$.
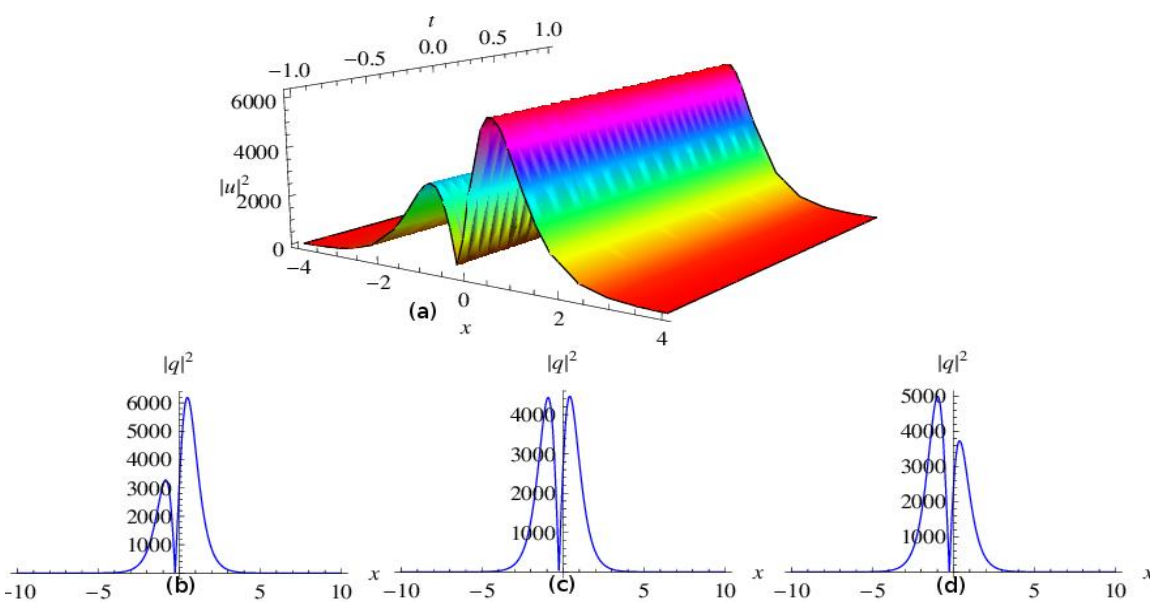

Figure 3: Soliton solution for localized inhomogeneity with a) $P_{2}=4$ b) $t=0$ c) $t=$ -27 and d) $t=-40$. 
Next we consider cubic type of inhomogeneity of the form $f=1+P_{3} x^{3}+P_{4} x^{2}$. Here it is noticed that for values of inhomogeneities $P_{3}<0.2$ and $P_{4}<1.2$, soliton nature is preserved. A further increase in the parameters $\left(P_{3}=0.5, P_{4}=1.9\right)$ leads to the formation of two humped solitons which interact with each other as time goes on. The behaviour is described in Figure 4(a). When the parameters are increased further to $P_{3}=P_{4}=5$, splitting of solitons takes place and two solitons become three solitons as described in Figure 4(b). Similar collision behaviour is also noticed. One soliton overtakes the other without any change in shape, amplitude and energy. Figures 4(c) and 4(d) illustrate the elastic collision between solitons. Similar behaviour is observed in the case of quadratic $f(x)=1+$ $P_{5} x^{2}+P_{6} x$ and periodic $f=1+P_{7} * \cos h(x)$ type inhomogeneities which is shown in Figures (5) and (6) respectively.
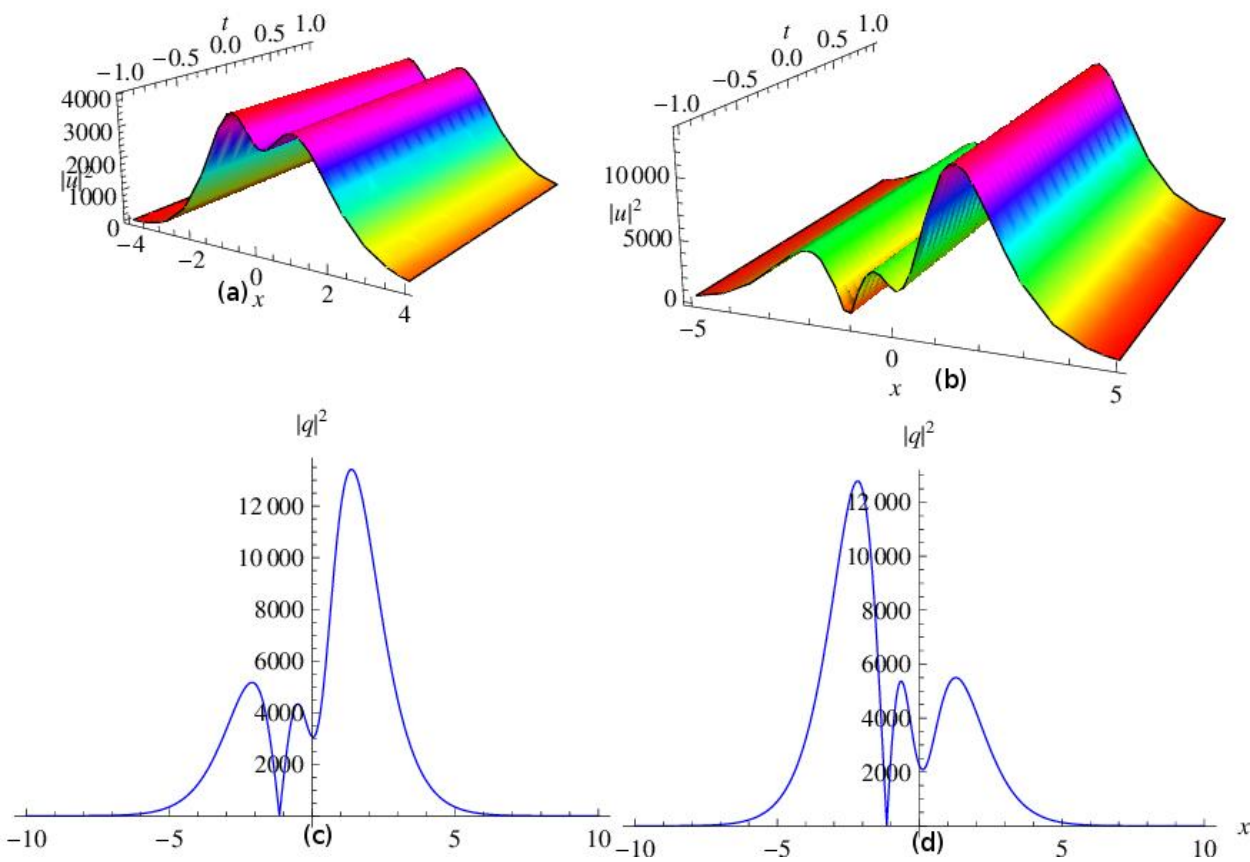

Figure 4: Soliton solution for cubic inhomogeneity with a) $P_{3}=0.5$ and $P_{4}=1.9$,

b) $P_{3}=P_{4}=5$, (c) $t=0$ and d) $t=-48$.
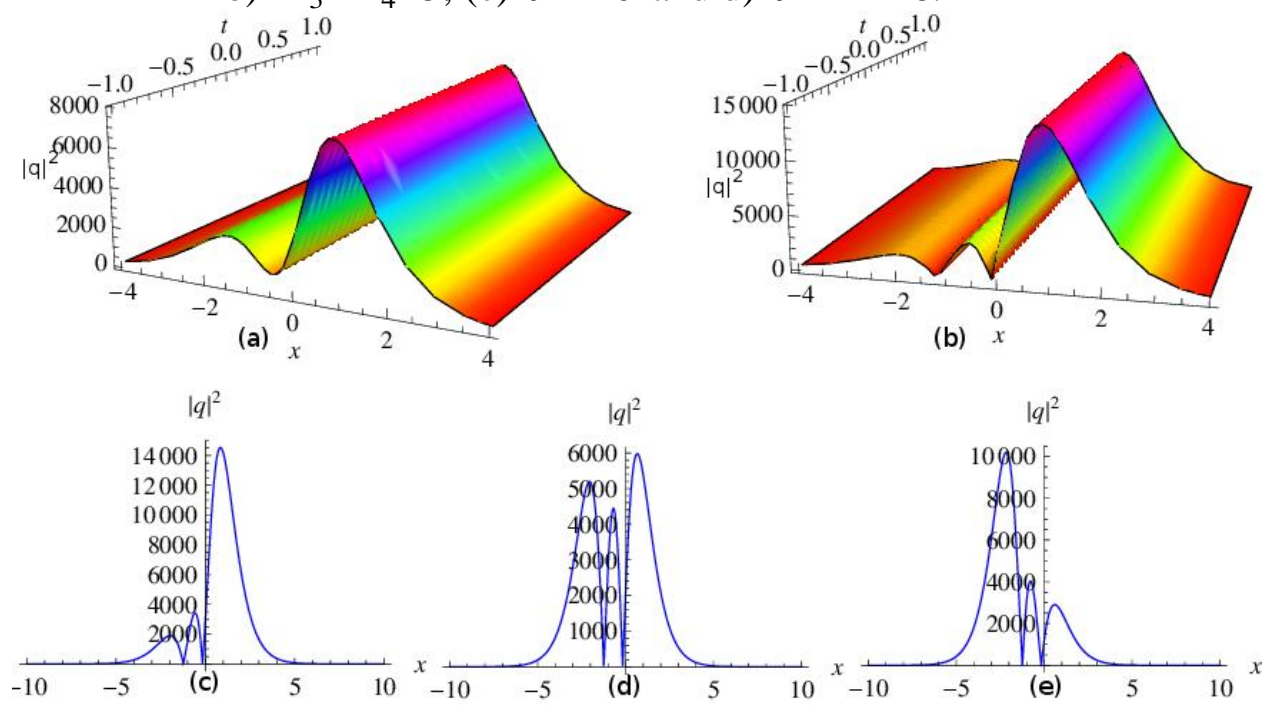

Figure 5: Soliton solution for quadratic inhomogeneity with a) $P_{5}=2.7$ and $P_{6}=3$

b) $\left.\left.P_{5}=P_{6}=8 \mathrm{c}\right) t=0 \mathrm{~d}\right) t=-27$ and e) $t=-47$. 

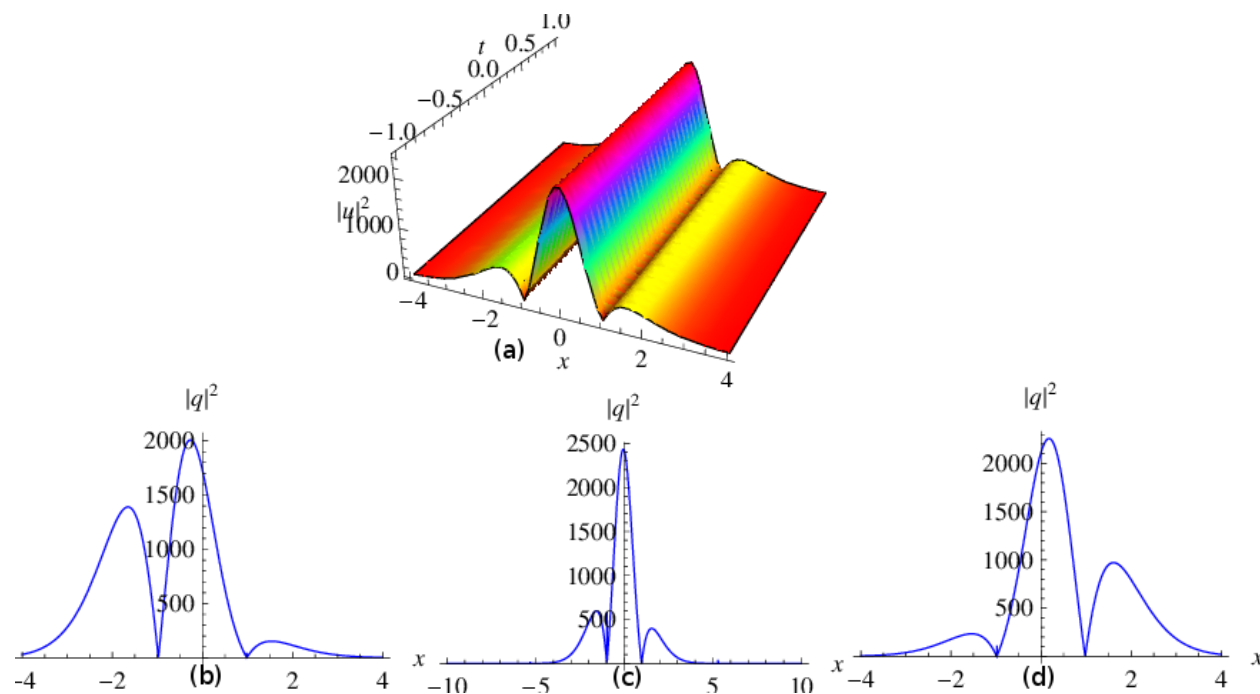

Figure 6: Soliton solution for Periodic inhomogeneity with a) $P_{7}=4$ b) $t=-50$, c)

$$
t=0 \text { and } \mathrm{d}) t=50 \text {. }
$$

In the biquadratic case $\left(f(x)=1+P_{8} x^{4}+P_{9} x^{2}\right)$ an interesting behaviour is noticed. For lower values of $P_{8}$ and $P_{9}$, we get three hump solitonic profile for the parameters $\left(P_{8}=1\right.$ and $\left.P_{9}=1.2\right)$ and the plot is described in Figure $7($ a) and for higher values the system is found to merge into two solitons. The interaction profile is described in Figure 7(c)-7(e).
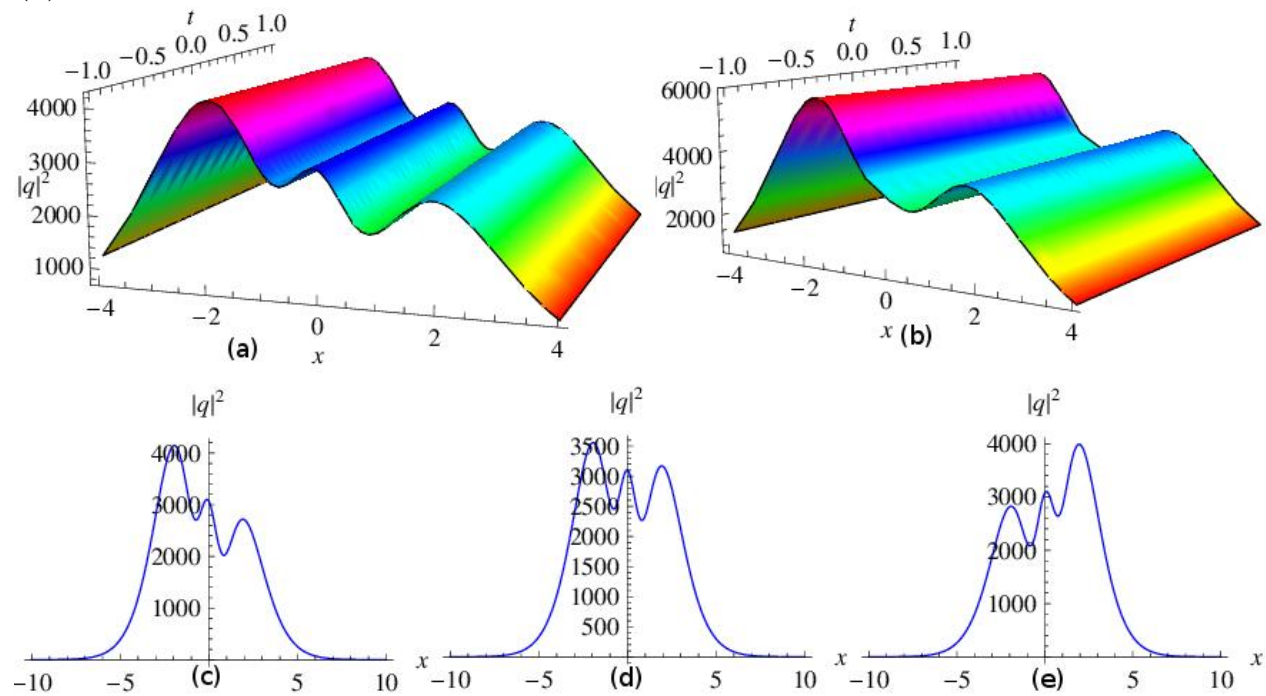

Figure 7: Soliton solution for bi-quadratic inhomogeneity with a) $P_{8}=1$ and $P_{9}=$ $1.2 \mathrm{c}) t=0 \mathrm{~d}) t=8$ and e) $t=20$.

\section{Conclusion}

In this paper, the nonlinear spin excitations in the form of solitons in a three dimensional inhomogeneous FM spin system with bilinear and anisotropy interactions are investigated in the semiclassical limit using the $\mathrm{H}-\mathrm{P}$ transformation combined with Glauber's coherent state representation. The dynamics is found to be governed by a $(3+1)$ dimensional PNLSE. The effect of inhomogeneity on the soliton is studied by carring out a perturbation analysis. We employ the Sine-Cosine function method to solve the three dimensional PNLSE with the aid of symbolic computation and the solution representing spin excitation is 
demonstrated graphically. We consider for our study various types of inhomogeneities such as linear, localized, cubic, quadratic, periodic and bi-quadratic. When the inhomogeneity parameter exceeds a certain value, one soliton starts splitting into two solitons which collide with each other but retaining its shape and amplitude after collision. For higher values of inhomogeneity, two soliton becomes three solitons interacting with each other elastically. But in the case of biquadratic inhomogeneity, for lower values we get three hump solitonic profile and for higher values the system is found to merge into two solitons.

\section{Appendix A}

$E_{i}, K_{i}, i=0,1 \ldots 7$ of Eq.(8) are given by

$$
\begin{aligned}
& E_{0}=\left[\left(-2 A+J^{\prime}+J^{\prime \prime}+J_{1}+J_{1}^{\prime}+J_{1}^{\prime \prime}+J_{2}\right) f_{i, j, k} a_{i, j, k} a_{i, j, k}^{\dagger}\right], \\
& E_{1}=\left[a_{i+1, j, k} a_{i+1, j, k}^{\dagger}-a_{i+1, j, k} a_{i, j, k}^{\dagger}-a_{i, j, k} a_{i+1, j, k}^{\dagger}\right] f_{i, j, k}, \\
& E_{2}=\left[a_{i, j+1, k} a_{i, j+1, k}^{\dagger}-a_{i, j+1, k} a_{i, j, k}^{\dagger}-a_{i, j, k} a_{i, j+1, k}^{\dagger}\right] f_{i, j, k}, \\
& E_{3}=\left[a_{i, j, k+1} a_{i, j, k+1}^{\dagger}-a_{i, j, k+1} a_{i, j, k}^{\dagger}-a_{i, j, k} a_{i, j, k+1}^{\dagger}\right] f_{i, j, k}, \\
& E_{4}=\left[a_{i+1, j+1, k} a_{i+1, j+1, k}^{\dagger}-a_{i+1, j+1, k} a_{i, j, k}^{\dagger}-a_{i, j, k} a_{i+1, j+1, k}^{\dagger}\right] f_{i, j, k}, \\
& E_{5}=\left[a_{i+1, j, k+1} a_{i+1, j, k+1}^{\dagger}-a_{i+1, j, k+1} a_{i, j, k}^{\dagger}-a_{i, j, k} a_{i+1, j, k+1}^{\dagger}\right] f_{i, j, k}, \\
& E_{6}=\left[a_{i, j+1, k+1} a_{i, j+1, k+1}^{\dagger}-a_{i, j+1, k+1} a_{i, j, k}^{\dagger}-a_{i, j, k} a_{i, j+1, k+1}^{\dagger}\right] f_{i, j, k}, \\
& E_{7}= \\
& {\left[a_{i+1, j+1, k+1} a_{i+1, j+1, k+1}^{\dagger}-a_{i+1, j+1, k+1} a_{i, j, k}^{\dagger}-a_{i, j, k} a_{i+1, j+1, k+1}^{\dagger}\right] f_{i, j, k} .}
\end{aligned}
$$

$$
K_{0}=\left[4 A a_{i, j, k}^{2}\right],
$$$$
K_{1}=
$$

$\left[-4 a_{i, j, k} a_{i+1, j, k} a_{i, j, k}^{\dagger} a_{i+1, j, k}^{\dagger}+a_{i, j, k} a_{i+1, j, k} a_{i, j, k}^{\dagger^{2}}+a_{i, j, k}^{2} a_{i, j, k}^{\dagger} a_{i+1, j, k}^{\dagger}+\right.$ $\left.a_{i+1, j, k}^{2} a_{i, j, k}^{\dagger} a_{i+1, j, k}^{\dagger}+a_{i, j, k} a_{i+1, j, k} a_{i+1, j, k}^{\dagger^{2}}\right] f_{i, j, k}$,

$$
K_{2}=
$$

$\left[-4 a_{i, j, k} a_{i, j+1, k} a_{i, j, k}^{\dagger} a_{i, j+1, k}^{\dagger}+a_{i, j, k} a_{i, j+1, k} a_{i, j, k}^{\dagger^{2}}+a_{i, j, k}^{2} a_{i, j, k}^{\dagger} a_{i, j+1, k}^{\dagger}+\right.$ $\left.a_{i, j+1, k}^{2} a_{i, j, k}^{\dagger} a_{i, j+1, k}^{\dagger}+a_{i, j, k} a_{i, j+1, k} a_{i, j+1, k}^{\dagger^{2}}\right] f_{i, j, k}$,

$$
K_{3}=
$$

$\left[-4 a_{i, j, k} a_{i, j, k+1} a_{i, j, k}^{\dagger} a_{i, j, k+1}^{\dagger}+a_{i, j, k} a_{i, j, k+1} a_{i, j, k}^{\dagger^{2}}+a_{i, j, k}^{2} a_{i, j, k}^{\dagger} a_{i, j, k+1}^{\dagger}+\right.$ $\left.a_{i, j, k+1}^{2} a_{i, j, k}^{\dagger} a_{i, j, k+1}^{\dagger}+a_{i, j, k} a_{i, j, k+1} a_{i, j, k+1}^{\dagger^{2}}\right] f_{i, j, k}$,

$$
K_{4}=\left[-4 a_{i, j, k} a_{i+1, j+1, k} a_{i, j, k}^{\dagger} a_{i+1, j+1, k}^{\dagger}+a_{i, j, k} a_{i+1, j+1, k} a_{i, j, k}^{\dagger^{2}}+\right.
$$

$\left.a_{i, j, k}^{2} a_{i, j, k}^{\dagger} a_{i+1, j+1, k}^{\dagger}+a_{i+1, j+1, k}^{2} a_{i, j, k}^{\dagger} a_{i+1, j+1, k}^{\dagger}+a_{i, j, k} a_{i+1, j+1, k} a_{i, j, k}^{\dagger^{2}}\right] f_{i, j, k}$, 
$K_{5}=\left[-4 a_{i, j, k} a_{i+1, j, k+1} a_{i, j, k}^{\dagger} a_{i+1, j, k+1}^{\dagger}+a_{i, j, k} a_{i+1, j, k+1} a_{i, j, k}^{\dagger^{2}}+\right.$ $\left.a_{i, j, k}^{2} a_{i, j, k}^{\dagger} a_{i+1, j, k+1}^{\dagger}+a_{i+1, j, k+1}^{2} a_{i, j, k}^{\dagger} a_{i+1, j, k+1}^{\dagger}+a_{i, j, k} a_{i+1, j, k+1} a_{i, j, k}^{\dagger^{2}}\right] f_{i, j, k}$,

$$
K_{6}=\left[-4 a_{i, j, k} a_{i, j+1, k+1} a_{i, j, k}^{\dagger} a_{i, j+1, k+1}^{\dagger}+a_{i, j, k} a_{i, j+1, k+1} a_{i, j, k}^{\dagger^{2}}+\right.
$$
$a_{i, j, k}^{2} a_{i, j, k}^{\dagger} a_{i, j+1, k+1}^{\dagger}+a_{i, j+1, k+1}^{2} a_{i, j, k}^{\dagger} a_{i, j+1, k+1}^{\dagger}+$ $\left.a_{i, j, k} a_{i, j+1, k+1} a_{i, j+1, k+1}^{\dagger^{2}}\right] f_{i, j, k}$,

$$
\begin{aligned}
& \quad K_{7}=\left[-4 a_{i, j, k} a_{i+1, j+1, k+1} a_{i, j, k}^{\dagger} a_{i+1, j+1, k+1}^{\dagger}+a_{i, j, k} a_{i+1, j+1, k+1} a_{i, j, k}^{\dagger^{2}}+\right. \\
& a_{i, j, k}^{2} a_{i, j, k}^{\dagger} a_{i+1, j+1, k}^{\dagger}+a_{i+1, j+1, k+1}^{2} a_{i, j, k}^{\dagger} a_{i+1, j+1, k+1}^{\dagger}+ \\
& \left.a_{i, j, k} a_{i+1, j+1, k+1} a_{i+1, j+1, k+1}^{\dagger^{2}}\right] f_{i, j, k} .
\end{aligned}
$$

\section{Appendix B}

$Q_{i}, R_{i}, i=0,1 \ldots 7$ of Eq.(10) are given by

$$
\begin{aligned}
& Q_{0}=2\left[A-\jmath-J^{\prime}-J^{\prime}-J_{1}-J_{1}^{\prime}-J_{1}^{\prime \prime}-J_{2}\right] f_{i, j, k}, \\
& Q_{1}=\left[u_{i, j, k} f_{i-1, j, k}-u_{i+1, j, k} f_{i, j, k}-u_{i-1, j, k} f_{i-1, j, k}\right], \\
& Q_{2}=\left[u_{i, j, k} f_{i, j-1, k}-u_{i, j+1, k} f_{i, j, k}-u_{i, j-1, k} f_{i, j-1, k}\right], \\
& Q_{3}=\left[u_{i, j, k} f_{i, j, k-1}-u_{i, j, k+1} f_{i, j, k}-u_{i, j, k-1} f_{i, j, k-1}\right], \\
& Q_{4}=\left[u_{i, j, k} f_{i-1, j-1, k}-u_{i+1, j+1, k} f_{i, j, k}-u_{i-1, j-1, k} f_{i-1, j-1, k}\right], \\
& Q_{5}=\left[u_{i, j, k} f_{i-1, j, k-1}-u_{i+1, j, k+1} f_{i, j, k}-u_{i-1, j, k-1} f_{i-1, j, k-1}\right], \\
& Q_{6}=\left[u_{i, j, k} f_{i, j-1, k-1}-u_{i, j+1, k+1} f_{i, j, k}-u_{i, j-1, k-1} f_{i, j-1, k-1}\right], \\
& Q_{7}=\left[u_{i, j, k} f_{i-1, j-1, k-1}-u_{i+1, j+1, k+1} f_{i, j, k}-u_{i-1, j-1, k-1} f_{i-1, j-1, k-1}\right] . \\
& R_{0}=\left[8 A\left|u_{i, j, k}\right|^{2} u_{i, j, k}\right],
\end{aligned}
$$

$$
R_{1}=
$$

$\left[-4\left|u_{i+1, j, k}\right|^{2} u_{i, j, k} f_{i, j, k}-4\left|u_{i-1, j, k}\right|^{2} u_{i, j, k} f_{i-1, j, k}+2\left|u_{i, j, k}\right|^{2} u_{i+1, j, k} f_{i, j, k}+\right.$ $u_{i, j, k}^{2} u_{i+1, j, k}^{*} f_{i, j, k}+\left|u_{i-1, j, k}\right|^{2} u_{i-1, j, k} f_{i-1, j, k}+\left|u_{i+1, j, k}\right|^{2} u_{i+1, j, k} f_{i, j, k}+$ $\left.u_{i, j, k}^{2} u_{i-1, j, k}^{*} f_{i-1, j, k}+2\left|u_{i, j, k}\right|^{2} u_{i-1, j, k} f_{i-1, j, k}\right]$,

$$
R_{2}=
$$

$\left[-4\left|u_{i, j+1, k}\right|^{2} u_{i, j, k} f_{i, j, k}-4\left|u_{i, j-1, k}\right|^{2} u_{i, j, k} f_{i, j-1, k}+2\left|u_{i, j, k}\right|^{2} u_{i, j+1, k} f_{i, j, k}+\right.$ $u_{i, j, k}^{2} u_{i, j+1, k}^{*} f_{i, j, k}+\left|u_{i, j-1}, k\right|^{2} u_{i, j-1, k} f_{i, j-1, k}+\left|u_{i, j+1, k}\right|^{2} u_{i, j+1, k} f_{i, j, k}+$ $\left.\left.u_{i, j, k}^{2} u_{i, j-1, k}^{*} f_{i, j-1, k}+2\left|u_{i, j, k}\right|^{2} u_{i, j-1, k} f_{i, j-1, k}\right)\right]$,

$$
R_{3}=
$$




$$
\begin{aligned}
& {\left[-4\left|u_{i, j, k+1}\right|^{2} u_{i, j, k} f_{i, j, k}-4\left|u_{i, j, k-1}\right|^{2} u_{i, j, k} f_{i, j, k-1}+2\left|u_{i, j, k}\right|^{2} u_{i, j, k+1} f_{i, j, k}+\right.} \\
& u_{i, j, k}^{2} u_{i, j, k+1}^{*} f_{i, j, k}+\left|u_{i, j, k-1}\right|^{2} u_{i, j, k-1} f_{i, j, k-1}+\left|u_{i+1, j, k}\right|^{2} u_{i, j, k+1} f_{i, j, k}+ \\
& \left.u_{i, j, k}^{2} u_{i, j, k-1}^{*} f_{i, j, k-1}+2\left|u_{i, j, k}\right|^{2} u_{i, j, k-1} f_{i, j, k-1}\right], \\
& \quad R_{4}=\left[-4 u_{i, j, k}\left(\left|u_{i+1, j+1, k}\right|^{2}+\left|u_{i-1, j-1, k}\right|^{2}\right)+2\left|u_{i, j, k}\right|^{2} u_{i+1, j+1, k}+\right. \\
& u_{i, j, k}^{2} u_{i+1, j+1, k}^{*}+\left|u_{i-1, j-1, k}\right|^{2} u_{i-1, j-1, k}+\left|u_{i+1, j+1, k}\right|^{2} u_{i+1, j+1, k}+ \\
& \left.u_{i, j, k}^{2} u_{i-1, j-1, k}^{*}+2\left|u_{i, j, k}\right|^{2} u_{i-1, j-1, k}\right], \\
& \quad R_{5}=\left[-4 u_{i, j, k}\left(\left|u_{i+1, j, k+1}\right|^{2}+\left|u_{i-1, j, k-1}\right|^{2}\right)+2\left|u_{i, j, k}\right|^{2} u_{i+1, j, k+1}+\right. \\
& u_{i, j, k}^{2} u_{i+1, j, k+1}^{*}+\left|u_{i-1, j, k-1}\right|^{2} u_{i-1, j, k-1}+\left|u_{i+1, j, k+1}\right|^{2} u_{i+1, j, k+1}+ \\
& \left.u_{i, j, k}^{2} u_{i-1, j, k-1}^{*}+2\left|u_{i, j, k}\right|^{2} u_{i-1, j, k-1}\right], \\
& \quad R_{6}=\left[-4 u_{i, j, k}\left(\left|u_{i, j+1, k+1}\right|^{2}+\left|u_{i, j-1, k-1}\right|^{2}\right)+2\left|u_{i, j, k}\right|^{2} u_{i, j+1, k+1}+\right. \\
& u_{i, j, k}^{2} u_{i, j+1, k+1}^{*}+\left|u_{i, j-1, k-1}\right|^{2} u_{i, j-1, k-1}+\left|u_{i, j+1, k+1}\right|^{2} u_{i, j+1, k+1}+ \\
& \left.u_{i, j, k}^{2} u_{i, j-1, k-1}^{*}+2\left|u_{i, j, k}\right|^{2} u_{i, j-1, k-1}\right], \\
& \quad R_{7}=\left[-4 u_{i, j, k}\left(\left|u_{i+1, j+1, k+1}\right|^{2}+\left|u_{i-1, j-1, k-1}\right|^{2}\right)+2\left|u_{i, j, k}\right|^{2} u_{i+1, j+1, k+1}+\right. \\
& u_{i, j, k}^{2} u_{i+1, j+1, k+1}^{*}+\left|u_{i-1, j-1, k-1}\right|^{2} u_{i-1, j-1, k-1}+\left|u_{i+1, j+1, k+1}\right|^{2} u_{i+1, j+1, k+1}+ \\
& \left.u_{i, j, k}^{2} u_{i-1, j-1, k-1}^{*}+2\left|u_{i, j, k}\right|^{2} u_{i-1, j-1, k-1}\right] .
\end{aligned}
$$

\section{References}

1. Saravanan M and Arnaudon A, Phys. Lett. A 07 (2018) 015

2. Rini Ganguly, Indranil Roy, Anurag Banerjee, Harkirat Singh, Amit Ghosal, and Pratap Raychaudhuril, Phys. Rev. B 96 (2017) 054509

3. Galkina E G and Ivanov B A, Low Temp Phys 44 (2018) 618

4. Mishin D D, Magnetic Materials 1991 (Vysshaya Shkola, Moscow) .

5. Hubert A and Schafer R, Magnetic Domains 1998 (Springer, Berlin).

6. Kavitha L and Daniel M, J. Phys. A: Math. Gen. 36 (2003) 10471

7. Daniel M and Kavitha L, Phys. Rev. B 63 ( 2001) 172302

8. Myrzakulov R, Daniel M and Amuda R, Physica A 234 (1997) 715

9. Daniel M and Amuda R, Phys. Rev. B 53 (1996) R2930

10. Daniel M and Beula J, Phys. Rev. B 77 (2008) 144416

11. Ameya Jagtap D and Vasudeva Murthy A S, Commun Nonlinear Sci Numer Simulat 64 (2018) 178

12. Latha M M and Christal Vasanthi C, Phys. Scr 89 (2014) 065204

13. Latha M M and Stally G A, j.cjph 56 (2018) 1021

14. Holstein T and Primakoff H, Phys. Rev. 58 (1940) 1098

15. Glauber R J, Phys. Rev. 131 (1963) 2766

16. Wazwaz A M, Math. Comput. Modelling 40 (2004) 499

17. Wazwaz A M, Appl. Math. Comput 159 (2004) 559 